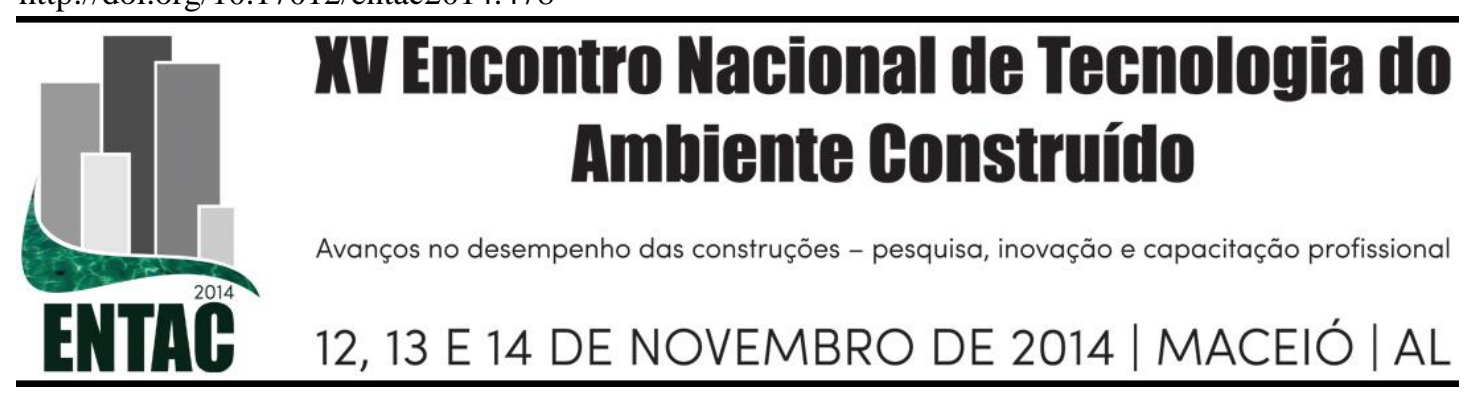

\title{
GESTÃO DO PROCESSO DE FLEXIBILIZAÇÃO E PERSONALIZAÇÃO DE PROJETOS
}

\author{
FERNANDES, Rosana da Silva (1); GONZÁLEZ, Marco Aurélio Stumpf (2)
}

(1) UNISINOS, e-mail: eng.rosanaf@gmail.com (2)UNISINOS, e-mail: mgonzalez@unisinos.br

\begin{abstract}
RESUMO
A flexibilização e a personalização de projetos são conceitos adotados pelas construtoras para se adequarem às exigências do mercado imobiliário, com atenção à satisfação dos usuários. No entanto, as estratégias estão sendo amplamente praticadas em todo o país, mas ainda não há uma gestão eficaz do processo de modificações. A participação dos usuários nas definições de projeto, característica da flexibilização e personalização, resulta em aumento da quantidade de intervenientes e informações. Assim, com o aumento do fluxo de dados, as falhas na comunicação e na retroalimentação do processo são ocorrências frequentes, necessitando de um sistema de gestão adequado à quantidade de informações. As falhas no fluxo de informações causam retrabalho, atrasos na fase de construção, aumento na geração de resíduos e perda de material. Diante disso, este trabalho tem como objetivo investigar os processos de flexibilização e personalização de projeto, em empreendimentos comercializados em Porto Alegre, RS. O desenvolvimento da pesquisa foi dividido em duas etapas: levantamento e análise da publicidade de 143 projetos; e quatro estudos de caso realizados em empresas de construção. Os resultados indicaram falhas na gestão das modificações de projeto, inclusive problemas no fluxo de informações. Com base na análise dos resultados, a pesquisa propõe um conjunto de diretrizes que incluem a utilização de TICs, tais como extranets de projeto, dispositivos móveis e plataforma BIM. As diretrizes visam à melhoria da gestão das modificações de projeto; buscando eficiência da comunicação entre os intervenientes, redução de retrabalho, diminuição dos resíduos gerados e retroalimentação de dados; agregando qualidade à edificação e maior satisfação dos usuários.
\end{abstract}

Palavras-chave: Modificações de projeto, Gestão da informação, TIC, BIM.

\begin{abstract}
The flexibility and customization of designs are concepts adopted by construction companies to suit the requirements of real estate market, with attention to user satisfaction. However, strategies are being widely practiced throughout the country, but there is still no effective management of change process. The participation of users in design decisions, characteristic of flexibility and customization, results in an increasing the amount of stakeholders and information. Thus, with the increased flow of data, failures in communication and process feedback are frequent occurrences, requiring an adequate management system to amount of information. The failures in the information flow cause rework, delays in the construction phase, increase in waste generation and loss of material. Thus, this study aims to investigate the processes of flexibility and customization in projects marketed in Porto Alegre, RS. The development of the research was divided into two phases: survey and analysis in advertising of 143 projects; and four case studies carried out in construction companies. The results indicate failures in managing the design changes, including problems in the information flow. Based on the analysis of the results, the research proposes a set of guidelines that include the use of ICTs, such as project extranets, mobile devices and BIM platform. The guidelines aim to improved management of the design changes; searching efficiency of communication between stakeholders, reduce rework, decrease of waste generated and feedback data; adding quality to the building and greater user satisfaction.
\end{abstract}

Keywords: Design changes, Information management, ICT, BIM. 


\section{INTRODUÇÃO}

Desde a década de 1990, são observadas mudanças na configuração dos empreendimentos no país, como projetos customizáveis, com alto grau de complexidade que mobilizam diferentes especialidades. Com isso, o setor da construção civil passou a buscar a eficiência com atenção às necessidades e satisfação dos usuários (ADESSE; MELHADO, 2003).

Conforme Santana, Oliveira e Meira (2007), a flexibilização e a personalização de projetos tornaram-se opções adotadas por construtoras para se adequarem às exigências do mercado imobiliário. Porém, ainda não existe uma gestão efetiva das modificações, resultando em retrabalhos, atrasos e desperdícios de materiais.

Segundo Larcher (2005), a flexibilização consiste no planejamento de modificações no projeto da edificação. Já a personalização baseia-se na possibilidade de atribuir ao produto características do consumidor, visando atender suas exigências (STAHL, 2005).

De acordo com Brandão (2011), as edificações frequentemente são projetadas considerando apenas as necessidades da ocupação inicial, causando dificuldades e custos adicionais para adaptar posteriormente o imóvel. Strapasson (2011) afirma que a falta de flexibilidade nos projetos ou a falta de atendimento às necessidades dos usuários podem causar intervenções, demolição parcial, e até a demolição completa de uma edificação.

Para Osmani, Glass e Price (2008), as alterações de requisitos dos usuários, durante a etapa de construção, é uma das principais causas da geração de resíduos em uma obra, além de problemas como detalhamento de projetos, falta de dados ou especificações inadequadas, e falta de comunicação entre os diversos intervenientes.

Para Santana, Oliveira e Meira (2007), o aumento do número de intervenientes e do volume de informações, são implicações de projetos com modificações que exigem uma gestão adequada para evitar a ocorrência de retrabalho. Stahl (2005) explica que a gestão das informações é importante para retroalimentação do projeto, para registro das alterações solicitadas e para armazenar dados sobre as modificações executadas na edificação.

Diante da importância do gerenciamento adequado do processo de modificações de projeto, incluindo os problemas relacionados à gestão do fluxo de informações, é objetivo deste trabalho investigar os processos de flexibilização e personalização de projetos em empreendimentos da cidade de Porto Alegre, RS.

A pesquisa inclui a identificação da situação da flexibilização e da personalização nos empreendimentos comercializados na região de estudo, formando um panorama da realidade do mercado imobiliário. Ainda, o trabalho abrange um estudo de casos para identificar os principais aspectos e problemas acarretados pela flexibilização e personalização de projetos, para identificação de problemas no processo de modificações e no fluxo de informações.

Com a análise dos resultados, foi elaborado um conjunto de diretrizes visando à melhoria do gerenciamento de empreendimentos com modificações de projeto. As diretrizes estão relacionadas aos principais problemas verificados durante a pesquisa, especialmente problemas relacionados à gestão do fluxo de informações. 


\section{REVISÃO BIBLIOGRÁFICA}

\subsection{Processo de projeto}

O processo de projeto na construção civil é complexo, devido às características dos atuais empreendimentos; composto por diversos subprojetos, desenvolvidos por diferentes profissionais de forma fragmentada e sequencial, gerando uma grande quantidade de informação (FABRICIO, 2007). Para Manzione e Melhado (2007), as falhas de projeto são causadas por problemas no atendimento dos requisitos dos usuários e pelas modificações de projeto executadas durante a fase de construção do empreendimento. Desta forma, Fabricio (2007) aponta a necessidade de uma coordenação eficiente de todo o processo, conciliando todos os envolvidos com o projeto e administrando o fluxo de informações. Ainda, conforme Brandão e Heineck (2007), no caso de empreendimentos com flexibilização e personalização de projetos, para a gestão eficiente das modificações, é necessário um amplo uso de Tecnologias de Informação e Comunicação (TICs) para a comunicação entre os intervenientes.

\subsection{Gestão da informação}

Em projetos com flexibilização e personalização, as frequentes alterações de projeto não são corretamente registradas e distribuídas a todos intervenientes, gerando erros na execução da obra (OLIVEIRA, 2005). Para Kiviniemi (2005) é preciso desenvolver métodos eficazes para gerenciar a grande quantidade de informações relacionadas aos requisitos dos usuários, através do uso de TICs. Krygiel e Nies (2008) citam o BIM (Building Information Modeling) para gerenciar a evolução do projeto e reduzir a quantidade de retrabalho gerado por erros de projeto. Para Eastman et al., (2011) o sistema BIM contribui para criação de um ambiente colaborativo. Para Crespo e Ruschel (2007) outra importante ferramenta é a extranet de projeto que consiste em um sistema colaborativo com comunicação e armazenamento de arquivos através da internet, possibilitando a coordenação centralizada das informações do projeto. Kuladinithi, Timm-Giel e Görg (2004) ressaltam, a computação móvel como laptops e smartphones, para comunicação entre os intervenientes e acesso ao projeto atualizado.

\section{FLEXIBILIZAÇÃO}

A flexibilização de projeto consiste na previsão e planejamento de alterações na planta da edificação (LARCHER, 2005). Conforme Brandão e Heineck (2007), o conceito de flexibilidade na construção civil surgiu na Europa, na década de 1970, em oposição à construção industrializada, utilizada para reconstrução de moradias em períodos pósguerra. No Brasil, o conceito de flexibilidade se consolidou na década de 1990, através do sistema de vendas de imóveis em planta, com a possibilidade de escolha da distribuição interna dos ambientes, entre vários layouts de planta. As principais formas de aplicação da flexibilidade são apresentadas por Brandão e Heineck (2007):

- Diversidade tipológica: opções de plantas, sem modificações;

- Flexibilidade propriamente dita: variabilidade de arranjos;

- Adaptabilidade: adaptação da função dos ambientes;

- Ampliabilidade: ampliação externa ou interna do imóvel;

- Junção/desmembramento: a divisão de um imóvel em dois ou o agrupamento de duas habitações adjacentes, formando uma só. 
Segundo Brandão e Heineck (2007), o entendimento das mudanças das necessidades dos usuários ainda na concepção do projeto, reduz a possibilidade de intervenções não planejadas, como demolições e adaptações da edificação. Entretanto, Larcher (2005) alerta sobre a possibilidade de aumento nos custos e da necessidade de ampliação dos prazos para execução das alterações de projeto. Ainda, conforme Brandão e Heineck (2007), a falta de flexibilidade e a falta de um profissional qualificado para execução das modificações, geralmente resulta em alterações onerosas e ineficientes.

\section{PERSONALIZAÇÃO}

A personalização baseia-se na possibilidade de atribuir características particulares do consumidor ao produto visando atender suas exigências (SANTANA; OLIVEIRA; MEIRA, 2007). Stahl (2005) aponta que a personalização é uma estratégia originada na indústria automobilística. Segundo Brandão e Heineck (2007), a personalização na construção civil começou a ser praticada na década de 1990, após a flexibilização, atendendo as exigências de clientes com maior poder aquisitivo; permitindo alterações na planta padrão e nos materiais de acabamento. Moschen (2003) apresenta uma classificação para os níveis de personalização praticados no mercado imobiliário:

- Personalização do arranjo dos espaços: eliminação, acréscimo ou alteração das dimensões de um ambiente;

- Alterações das funções dos espaços: alteração de uso ou função dos espaços;

- Personalização das instalações (hidráulica, elétrica e condicionamento de ar): acréscimo, eliminação ou alteração da localização dos pontos, vinculadas à personalização do arranjo ou à alteração de função dos espaços;

- Alteração dos materiais de revestimentos e acabamentos: personalização dos acabamentos, devido à variedade de modelos e às novidades do mercado.

Para Brandão (2011), a interferência do cliente no projeto torna o processo construtivo ainda mais complexo, exigindo uma gestão eficiente do processo de personalização para diminuir a ocorrência de retrabalho, aumento de custos e atrasos no cronograma da obra. Conforme Santana, Oliveira e Meira (2007), as maiores dificuldades estão relacionadas à sequência dos serviços na obra, os prazos para modificações e o aumento do número de intervenientes. Desta forma, Stahl (2005) aponta o uso das TICs para uma melhor gestão das informações, permitindo uma atualização mais ágil dos projetos e documentos, reduzindo o retrabalho e a quantidade de resíduos.

\section{MÉTODO}

A pesquisa foi desenvolvida em duas etapas. Na primeira etapa foi realizado um levantamento de dados para identificação da situação do mercado imobiliário em relação à ocorrência de flexibilização e/ou personalização de imóveis e, em relação às modificações mais comercializadas e permitidas pelas empresas. Na segunda etapa foi realizada uma pesquisa para identificar os principais aspectos e problemas acarretados pela flexibilização e personalização de projetos, utilizando o método de estudo de casos.

A etapa de identificação da situação da oferta de modificações de projeto foi realizada através da coleta e análise da publicidade de 143 empreendimentos formando um panorama da realidade do mercado imobiliário. A publicidade dos empreendimentos foi coletada na mídia, em anúncios em jornais, folders, sites de construtoras, incorporadoras e imobiliárias, além de visitas a feiras de imóveis e visitas aos plantões de venda. 
Os empreendimentos foram classificados em flexíveis, ou seja, com opção de escolha do layout de planta; e personalizáveis, quando permitia modificações nos materiais de acabamento e alterações nas instalações do imóvel. Esta classificação permitiu a formação de um panorama do mercado imobiliário: identificação da estratégia de venda mais ofertada - flexibilização ou personalização - além da identificação dos layouts mais comercializados e das modificações mais ofertadas pelas empresas; determinando as maiores dificuldades na gestão das modificações.

$\mathrm{Na}$ etapa de estudos de casos foram investigados: o desenvolvimento do processo de modificações de projeto, a coordenação dos projetos, a troca de informações entre os intervenientes, os softwares utilizados pelas empresas, as modificações planejadas no projeto, as alterações mais solicitadas pelos usuários, os prazos para modificações e os principais problemas relacionados às alterações de projeto.

Para coleta de dados a respeito do processo de projeto, produção, troca de informações e gerenciamento das modificações de projeto em cada empresa, foram realizadas entrevistas estruturadas com os profissionais envolvidos no processo, como os arquitetos, engenheiros ou coordenadores de projetos. Também foram realizadas visitas às obras dos empreendimentos com possibilidade de modificações de projeto, para observação do processo de produção e análise da troca de informações entre os intervenientes do processo, permitindo identificar problemas recorrentes nas empresas construtoras.

Com a análise dos resultados, foi elaborado um conjunto de diretrizes visando à melhoria do gerenciamento de empreendimentos com flexibilização ou personalização de projeto. As diretrizes estão relacionadas aos principais problemas verificados durante a pesquisa, especialmente problemas relacionados à gestão das informações.

\section{RESULTADOS E DISCUSSÃO}

\subsection{Situação da oferta de modificações de projeto}

De acordo com os resultados, observou-se a maior ocorrência da comercialização de empreendimentos com customização, onde as empresas ofertavam a flexibilização diferentes layouts de projeto - em conjunto com a personalização - modificações de materiais de acabamento.

A partir da análise dos dados, observou-se que na flexibilização, além da planta padrão de três dormitórios são oferecidas mais opções de layouts de plantas. Na flexibilização, os layouts de projeto mais ofertados são (em ordem): a cozinha americana; o aumento da sala de estar e a criação de um closet, ambos pela eliminação do terceiro dormitório.

Em relação à personalização, os resultados apontaram que as empresas comercializam imóveis que oferecem a flexibilização em conjunto com a personalização do projeto, com opções de layouts alternativos e a possibilidade de modificações em acabamentos e instalações. Na personalização, verificou-se que as modificações mais solicitadas são (em ordem): pisos, revestimentos, instalações, alterações de layout, metais, louças, bancadas e instalação de banheira.

Na Figura 1, um exemplo da publicidade de venda de apartamentos com flexibilização, com demonstração do projeto original e das opções de layout: no projeto original há três dormitórios (a) e nas outras opções o terceiro dormitório é modificado em diferentes situações, para aumento da sala de estar (b) ou criação de um closet (c,d). 
Figura 1 - Publicidade de empreendimento com flexibilização de projeto (a,b,c,d)

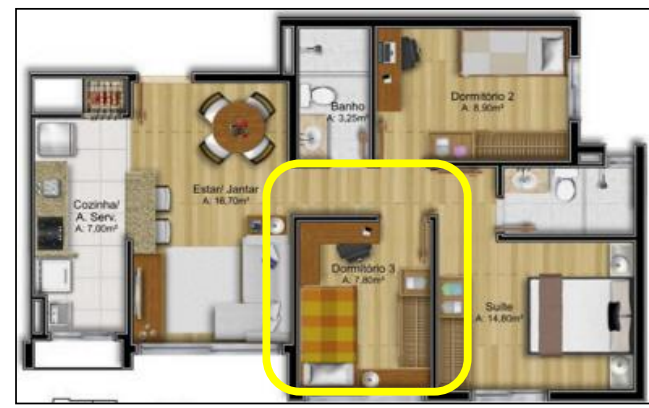

a) Projeto original

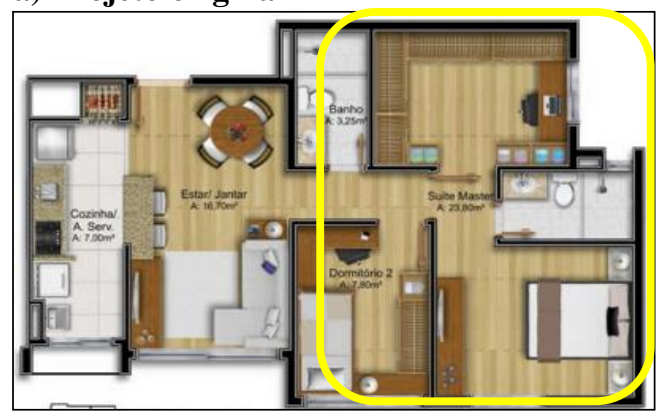

c) Projeto modificado: closet

Fonte: Divulgação da empresa

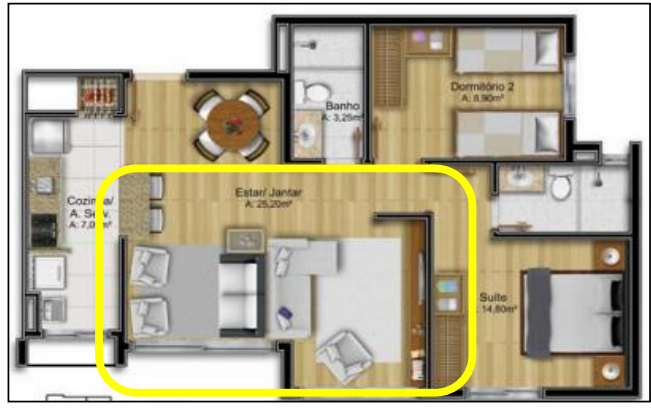

b) Projeto modificado: aumento da sala

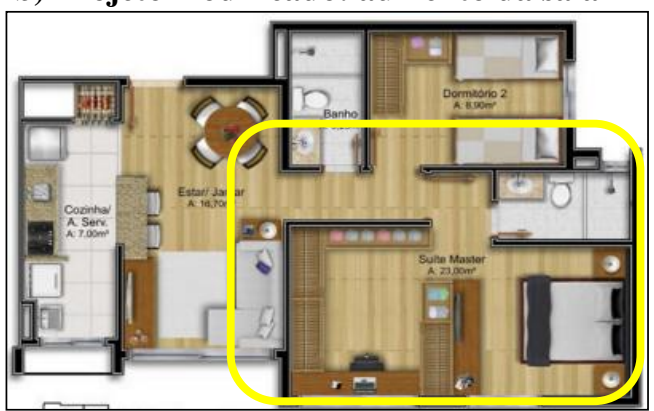

d) Projeto modificado: closet

Na Figura 2 é demonstrada uma interface para personalizar o projeto de um imóvel, simulando as modificações requisitadas pelo usuário. Neste empreendimento são ofertados diferentes layouts de projeto - flexibilização - e diversas formas de personalizar o imóvel, como inclusão de lavabo e até modificação de pisos.

\section{Figura 2 - Publicidade de empreendimento: interface para personalizar o projeto}

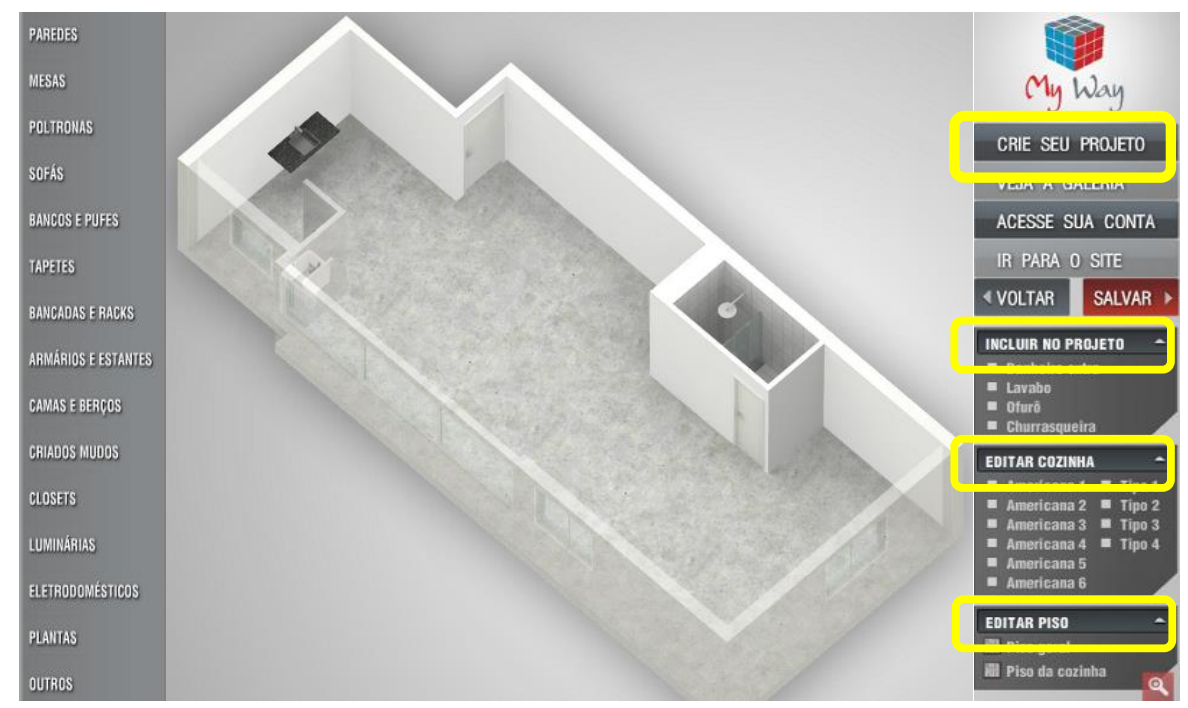

Fonte: Divulgação da empresa

Diante dos resultados, verificou-se que cada empresa permite determinadas modificações de projeto. No entanto, observou-se que a maioria das empresas permitia a 
escolha de layouts mesmo após a execução das paredes. De acordo com a publicidade dos empreendimentos, não é estipulado um prazo máximo para alterações, provocando modificações durante a fase de construção. Estas modificações podem causar demolições, para serviços já executados, além da geração de resíduos, retrabalho e até atrasos no cronograma.

Ainda, considerando a complexidade dos atuais projetos e a ampliação da oferta destes empreendimentos, incluindo edificações para a classe média baixa - com grande número de apartamentos - é demonstrada a importância da gestão das modificações, principalmente a gestão da troca de informações entre os intervenientes.

\subsection{Estudo de casos}

\section{Tabela 1 - Estudo de casos}

\begin{tabular}{|c|c|c|c|c|}
\hline Questão & Empresa 1 & Empresa 2 & Empresa 3 & Empresa 4 \\
\hline $\begin{array}{l}1 . \\
\text { Coordenação } \\
\text { dos projetos }\end{array}$ & $\begin{array}{l}\text { Gerente e Setor de } \\
\text { personalização }\end{array}$ & $\begin{array}{l}\text { Setor e gerente de } \\
\text { projetos }\end{array}$ & $\begin{array}{l}\text { Sem coordenação; } \\
\text { responsável - autor } \\
\text { do projeto }\end{array}$ & $\begin{array}{l}\text { Gerente e Setor de } \\
\text { personalização }\end{array}$ \\
\hline $\begin{array}{l}2 . \\
\text { Troca de } \\
\text { informações }\end{array}$ & $\begin{array}{l}\text { E-mail, reuniões, } \\
\text { telefone, extranet; } \\
\text { obra- laptops com } \\
\text { internet sem fio }\end{array}$ & $\begin{array}{l}\text { E-mail, reuniões, } \\
\text { telefone, extranet; } \\
\text { obra- netbooks com } \\
\text { internet sem fio }\end{array}$ & $\begin{array}{l}\text { E-mail, reuniões, } \\
\text { telefone, Software } \\
\text { gestão; laptops com } \\
\text { internet sem fio }\end{array}$ & $\begin{array}{l}\text { E-mail, reuniões, } \\
\text { telefone, extranet; } \\
\text { obra- laptops com } \\
\text { internet sem fio }\end{array}$ \\
\hline $\begin{array}{l}3 . \\
\text { Softwares }\end{array}$ & $\begin{array}{l}\text { Auto CAD; } \\
\text { MS Project; } \\
\text { SketchUp }\end{array}$ & \begin{tabular}{|l|} 
Auto CAD; \\
MS Project
\end{tabular} & $\begin{array}{l}\text { Auto CAD; } \\
\text { MS Project; } \\
\text { Software de gestão } \\
\text { com acesso internet }\end{array}$ & $\begin{array}{l}\text { Auto CAD; } \\
\text { MS Project; } \\
\text { Software de } \\
\text { maquetes virtuais }\end{array}$ \\
\hline $\begin{array}{l}4 . \\
\text { Flexibilização }\end{array}$ & $\begin{array}{l}\text { Oferta de } \\
\text { diferentes layouts }\end{array}$ & $\begin{array}{l}\text { Oferta de } \\
\text { diferentes layouts }\end{array}$ & $\begin{array}{l}\text { Oferta de diferentes } \\
\text { layouts }\end{array}$ & $\begin{array}{l}\text { Oferta de diferentes } \\
\text { layouts }\end{array}$ \\
\hline $\begin{array}{l}5 . \\
\text { Personalização }\end{array}$ & $\begin{array}{l}\text { Acabamentos e } \\
\text { instalações }\end{array}$ & \begin{tabular}{|l} 
Alguns \\
acabamentos
\end{tabular} & $\begin{array}{l}\text { Acabamentos e } \\
\text { instalações }\end{array}$ & $\begin{array}{l}\text { Acabamentos e } \\
\text { instalações }\end{array}$ \\
\hline \begin{tabular}{l|} 
6. Principais \\
modificações \\
solicitadas \\
pelos \\
compradores \\
\end{tabular} & $\begin{array}{l}\text { Revestimentos; } \\
\text { pisos; metais; } \\
\text { instalações; } \\
\text { banheira }\end{array}$ & \begin{tabular}{|l|} 
Aumento da sala \\
de estar e closet; \\
cozinha americana; \\
revestimentos; \\
pisos; metais \\
\end{tabular} & $\begin{array}{l}\text { Aumento da sala } \\
\text { de estar; cozinha } \\
\text { americana; } \\
\text { revestimentos; } \\
\text { pisos; instalações }\end{array}$ & $\begin{array}{l}\text { Aumento da sala } \\
\text { de estar; } \\
\text { revestimentos; } \\
\text { pisos; metais; } \\
\text { instalações } \\
\end{array}$ \\
\hline $\begin{array}{l}\text { 7. Prazo para } \\
\text { autorização de } \\
\text { modificações }\end{array}$ & $\begin{array}{l}\text { Depende de } \\
\text { aprovação do setor }\end{array}$ & $\begin{array}{l}\text { Antes da execução } \\
\text { dos revestimentos } \\
\text { internos }\end{array}$ & $\begin{array}{l}\text { Até } 6 \text { meses antes } \\
\text { do habite-se }\end{array}$ & $\begin{array}{l}\text { Depende de } \\
\text { aprovação do } \\
\text { setor de projetos }\end{array}$ \\
\hline $\begin{array}{l}8 . \mathrm{N}^{\mathrm{o}} \mathrm{de} \\
\text { unidades } \\
\text { modificadas }\end{array}$ & $80 \%$ personalização & $\begin{array}{l}90 \% \text { flexibilização } \\
50 \% \text { personalização }\end{array}$ & $\begin{array}{l}50 \% \text { flexibilização } \\
70 \% \text { personalização }\end{array}$ & $\begin{array}{l}40 \% \text { flexibilização } \\
70 \% \text { personalização }\end{array}$ \\
\hline $\begin{array}{l}\text { 9. Problemas } \\
\text { com as } \\
\text { modificações } \\
\text { de projetos }\end{array}$ & $\begin{array}{l}\text { Atrasos de } \\
\text { cronograma e } \\
\text { entrega de } \\
\text { materiais; } \\
\text { retrabalho; perda } \\
\text { de material } \\
\text { aprox. } 10 \%\end{array}$ & \begin{tabular}{|l} 
Gestão da \\
informação; \\
atrasos; estocagem \\
de novos materiais; \\
retrabalho; perda \\
de material \\
aprox.10\%
\end{tabular} & $\begin{array}{l}\text { Gestão das } \\
\text { modificações; } \\
\text { falhas de projeto e } \\
\text { execução; } \\
\text { retrabalho; perda } \\
\text { de material } \\
\text { aprox. } 20 \%\end{array}$ & $\begin{array}{l}\text { Falhas de } \\
\text { comunicação; } \\
\text { falhas de projeto e } \\
\text { execução; } \\
\text { retrabalho; perda } \\
\text { de material de } \\
\text { aprox. } 15 \%\end{array}$ \\
\hline
\end{tabular}

Fonte: Elaborada pelos autores 
De acordo com as respostas obtidas nas entrevistas e na observação do andamento das obras, verificou-se que a empresa 1 possui um coordenador de projetos e um setor de personalização; e os softwares utilizados não permitem a integração do projeto e produção, gerando retrabalho e atrasos na execução. No caso da empresa 2, são utilizados meios tradicionais de comunicação sem softwares de gestão, observando-se a falta de integração entre os envolvidos; causando retrabalho, perdas e atrasos na produção. Em relação à empresa 3, não há um controle específico para as modificações; a troca de informações ocorre de maneira informal devido a falta de softwares de gestão, causando problemas de retrabalho e perdas, relatados na entrevista. Por último, a empresa 4 possui um gerenciamento das modificações através de um setor específico e de um gerente de projetos, além da utilização de uma extranet de projeto, mas ainda foram constatados problemas devido a falta de informações dos projetos personalizados.

Conforme análise, as empresas possuem métodos diferentes para gerenciar os projetos. Porém, estes métodos se mostraram insuficientes para a demanda de modificações observada; com problemas de retrabalho, perda de material e atrasos. Também, notou-se a falta de TICs e BIM, para integração do projeto com a produção, pois a maioria das empresas possui um processo de projeto tradicional, sem uma efetiva colaboração entre os intervenientes. Outra questão observada é a dificuldade de gerenciamento de empreendimentos com grande número de apartamentos com modificações de projeto; percebendo-se a importância da gestão adequada ao grande volume de informações.

Além disso, outros fatores mostraram-se relevantes para a gestão do processo, tais como, prazos rígidos para as modificações, além de limites para personalização; pois foi detectada a relação da ocorrência de retrabalho e perda de material quando estas questões não eram controladas com rigor.

Desta forma, com a formação de um panorama do mercado imobiliário e com a identificação dos métodos de gestão nas empresas do estudo de casos; este trabalho propõe diretrizes para a melhoria da gestão das modificações de projeto, principalmente para o fluxo de informação, onde foram observados os maiores problemas de gestão das modificações.

\subsection{Diretrizes para gestão de projetos com modificações}

Como apresentado, no processo de modificações de projeto há diversos intervenientes. Além disso, o projeto ainda é tratado de forma sequencial, com subdivisão dos projetos, gerando uma grande quantidade de informação. Estes fatores ressaltam a necessidade da gestão adequada das informações. Nas empresas pesquisadas, a utilização de TICs é indispensável para comunicação entre os intervenientes.

De acordo com a literatura, através da gestão da informação, podem-se minimizar problemas de retrabalho, desperdício de materiais e mão de obra, além de atrasos de cronograma e aumento do custo da edificação; problemas recorrentes nos casos estudados. Ainda, com a utilização de TICs é possível controlar a execução das alterações no canteiro de obras, com uma atualização mais ágil dos projetos, reduzindo falhas na troca de informações.

Assim, com base nos resultados obtidos, é proposto um conjunto de cinco diretrizes visando à melhoria da gestão das modificações, através do gerenciamento das informações e da gestão do processo. Trata-se de diretrizes aplicáveis para os casos apresentados, bem como, para as demais empresas que oferecem a possibilidade modificações durante a execução dos projetos. As diretrizes possuem ênfase na comunicação entre os intervenientes. A seguir, são descritas as diretrizes propostas: 
- Promover a eficiência da comunicação entre os intervenientes: uso de dispositivos móveis com Internet sem fio, como: laptops, netbooks e tablets. Assim, obtém-se uma comunicação ágil, com dados atualizados e acessíveis à distância.

- Promover a gestão do fluxo de informações: uso de extranets de projeto e softwares de gestão para reduzir problemas de comunicação entre o setor de projetos e a obra, além de problemas de retrabalho e perda de material.

- Integrar as informações de projeto e produção: implantação de sistemas BIM para integração das informações em um único arquivo digital, facilitando as atualizações e a gestão do projeto; permitindo a redução de problemas na compra de materiais, com atualização simultânea de quantitativos.

- Estabelecer a coordenação de projetos com modificações: implantação de setor para gerenciar as modificações, e definição de um coordenador de projetos. Assim, pode-se garantir a qualidade do projeto com soluções adequadas, evitando o retrabalho; estimular a colaboração entre os intervenientes, centralizar a tomada de decisões, e controlar o fluxo de informações.

- Definir procedimentos para gestão das modificações: definição de limites para as modificações (prazo para solicitação e lista de itens personalizáveis), além do planejamento da execução das modificações.

\section{CONCLUSÕES}

A participação do usuário nas definições de projeto já é uma estratégia integrada às ofertas do mercado imobiliário, sendo considerado um diferencial de venda. A flexibilização e a personalização agregam qualidade ao empreendimento, além de proporcionar maior satisfação do usuário; permitindo adequar o projeto às suas necessidades, reduzindo a ocorrência de reformas realizadas durante o ciclo de vida das edificações. Além disso, com a adequação da edificação às necessidades dos usuários, reduz-se a ocorrência de desperdícios durante as reformas. Os desperdícios e perdas ocorrem pela falta de flexibilidade do projeto original e pela falta de um profissional qualificado para as alterações.

De acordo com o estudo, as maiores dificuldades enfrentadas pelas empresas estão relacionadas ao grande fluxo de informações, com aumento dos dados de projeto e aumento do número de intervenientes. Ainda, a falta de integração entre os intervenientes, a falta de coordenação de projetos com modificações e a falta de investimentos em TICs e BIM; são fatores que contribuem para ocorrência de erros de projeto, retrabalho, atrasos e perda de material. Estas falhas são causadoras de problemas no processo de projeto e produção, observados durante o acompanhamento das obras estudadas. Desta forma, o conjunto de diretrizes proposto, visa à eficiência da gestão da comunicação entre os intervenientes e a redução do retrabalho, proporcionando a melhoria da gestão de projetos com modificações.

\section{REFERÊNCIAS}

ADESSE, E.; MELHADO, S. B. A coordenação de projetos externa em empresas construtoras e incorporadoras de pequeno e médio portes. In: WORKSHOP BRASILEIRO DE GESTÃO DO PROCESSO DE PROJETO NA CONSTRUÇÃO DE EDIFÍCIOS, 3., 2003, Belo Horizonte.

Anais... Belo Horizonte: UFMG, 2003. 
BRANDÃO, D. Q. Disposições técnicas e diretrizes para projeto de habitações sociais evolutivas. Ambiente Construído, Porto Alegre, v. 11, n. 2, jun. 2011.

BRANDÃO, D. Q.; HEINECK, L. F. M. Estratégias de flexibilização de projetos residenciais iniciadas na década de 1990 no Brasil: tão somente um recurso mercadológico? Ambiente Construído, Porto Alegre, v. 7, n. 4, p. 71-87, out./dez. 2007.

CRESPO, C. C.; RUSCHEL R. C. Solução BIM para a melhoria no processo de projeto. In: SIMPÓSIO BRASILEIRO DE GESTÃO E ECONOMIA DA CONSTRUÇÃO, 2007a Campinas, Anais... Campinas: V SIBRAGEC, 2007.

EASTMAN, C.; TEICHOLZ, P.; SACKS, R.; LISTON, K. BIM Handbook: A Guide to Building Information Modeling for Owners, Managers, Designers, Engineers, and Contractors. Hoboken, New Jersey: John Wiley \& Sons, 2011. 626 p.

FABRICIO, M. M. O arquiteto e o coordenador de projetos. Pós - Revista Programa PósGraduação Arquitetura e Urbanismo da FAUUSP, n. 22, p. 26-50, dez 2007.

KIVINIEMI, A. Requirements Management Interface to Building product Models. 2005. $343 \mathrm{f}$. Thesis (Doctor of Philosophy) - Department of Civil and Environmental Engineering Center for Integrated Facility Engineering, Stanford University, 2005.

KRYGIEL, E.; NIES, B. Green BIM: successful sustainable design with Building Information Modelling. Indianapolis: Wiley Publishing, Inc., 2008. 266 p.

KULADINITHI, K.; TIMM-GIEL, A.; GÖRG, C. Mobile ad-hoc communications in AEC industry. Electronic Journal of Information Technology in construction - Itcon, v. 9, p. 313-323, ago 2004.

LARCHER, J. V. M. Diretrizes visando à melhoria de projetos e soluções construtivas na expansão de habitações de interesse social. 2005. 119f. Dissertação (Mestrado em Engenharia Civil) - Universidade Federal do Paraná, Curitiba, PR, 2005.

MANZIONE, L.; MELHADO, S. B. Porque os Projetos atrasam? Uma análise crítica da ineficácia do planejamento de projetos adotada no mercado imobiliário de São Paulo. In: ENCONTRO DE TECNOLOGIA DA INFORMAÇÃO E COMUNICAÇÃO NA CONSTRUÇÃO CIVIL, 3., 2007, Porto Alegre. Anais... Porto Alegre: UFRGS, 2007.

MOSCHEN, P. D. C. Uma Metodologia para Personalização de Unidades Habitacionais em Empreendimentos Imobiliários Multifamiliares. 2003. 212f. Dissertação. (Mestrado em Engenharia de Produção) - Programa de Pós- Graduação em Engenharia de Produção, Universidade Federal de Santa Catarina, Florianópolis, SC, 2003.

OLIVEIRA, G.G. Coordenação de projetos de obras de edificação: proposta de ferramenta computacional para programação e controle do fluxo de informações com uso de sistema colaborativo. 2005. Dissertação (Mestrado em Engenharia) - Programa de Pós- Graduação em Engenharia Civil. Universidade Federal do Rio Grande do Sul, Porto Alegre. 2005.

OSMANI, M.; GLASS, J.; PRICE, A. Architects' perspectives on construction waste reduction by design. Waste Management, v. 28, p. 1147-1158, 2008.

SANTANA, C.; OLIVEIRA, D.; MEIRA, A. Personalização de imóveis verticais residenciais: um estudo exploratório nas construtoras da cidade de João Pessoa. In: CONGRESSO DE PESQUISA E INOVAÇÃO DA REDE NORTE NORDESTE DE EDUCAÇÃO TECNOLÓGICA JOÃO PESSOA, 2., 2007, João Pessoa. Anais... João Pessoa: Cefet/PB, 2007.

STAHL, L. M. A customização em massa no mercado imobiliário de Londrina: um estudo multicaso. 2005. 115f. Dissertação (Mestrado em Administração) - Programa de PósGraduação em Administração, Universidade Estadual de Londrina, Londrina, PR, 2005.

STRAPASSON, D. C. Flexibilidade em projetos de edificações de ensino superior: estudo de caso na UFPR. 2011. Dissertação (Mestrado em Engenharia Civil) - Programa de PósGraduação em Construção Civil, Universidade Federal do Paraná, Curitiba, PR, 2011. 